A Case Report

\title{
ONCHOCERCIASIS: A NEGLECTED TROPICAL DISEASE STILL WITHIN AND AROUND US: A CASE REPORT FROM MAKURDI, NORTH-CENTRAL NIGERIA
}

Echekwube P.O, * Abidakun O.A.

Department of Medicine, College of Health Sciences, Benue State University, Makurdi, Nigeria.

*Corresponding Author: Dr Patrick Echekwube, Department of Medicine, College of Health Sciences, Benue State University, Makurdi, Nigeria.

Published: November $23^{\text {rd }} 2018$

\section{Abstract}

Onchocerciasis is a parasitic disease caused by the filarial worm Onchocerca volvulus. The disease is one of the Neglected Tropical Diseases which causes various cutaneous and extra-cutaneous disorders and creates a heavy burden on the sufferers. There is ongoing transmission of the disease despite all efforts at elimination mostly in rural communities. We report a case in a farmer who presented with features of chronic onchocerciasis and was further stigmatized in his community. Therefore, all efforts at elimination of the disease should be intensified.

Keywords: Makurdi, Neglected Tropical Disease, Onchocerciasis.

\section{Introduction}

Onchocerciasisisis a parasitic disease caused by the filarial worm Onchocerca volvulus. Humans acquire onchocerciasis through the bite of Simulium blackflies. The blackflies develop and breed in fast-flowing rivers and streams, mostly in remote villages located near fertile land where people rely mainly on agriculture for a living. In the human host, the adult worms live in subcutaneous tissue and produce microfilariae, which are found in the skin and eye and cause various cutaneous and ocular symptoms. ${ }^{1}$ The World Health Organization refers to Neglected Tropical Diseases (NTDs) as a diverse group of communicable diseases that prevail in the tropical and subtropical regions of the world. ${ }^{2}$ These diseases thrive among populations living in poverty, without adequate sanitation and in close contact with infectious vectors and domestic animals. The NTDs are prevalent in about 149 countries including Nigeria and these diseases affect more than one billion people and cost developing economies billions of dollars every year. ${ }^{1,2}$ There has been lots of effort at the elimination of onchocerciasis in Nigeria ${ }^{3}$ but there are still undetected cases which often present, quite unfortunately, at late stages to the hospitals where they can get expertise care and thus are worth reporting for improved surveillance and management. The early detection and management of onchocerciasis is key because the disease, in its chronic/late stages, could result in unbearable cutaneous and ocular symptoms such as persistent pruritus and visual impairment respectively which would prevent affected individuals from fending for themselves and further worsen their poverty.

\section{Case Report}

We report a case of a 60 year old male farmer, who hails from and resides at Aliade, located about 60 kilometers from Makurdi, the Benue state capital. He was referred from a Missionary hospital to the Benue State University Teaching Hospital, Makurdi on account of generalized skin eruptions and pruritus for 10 years.

The eruptions were initially at the extremities before spreading to the face and trunk. The initial eruptions were said to be erythematous and pruritic and patient had been scratching the lesions since their onset yet with little or no relief. He was fond of taking his bath and swimming in the fast flowing rivers close to his farmlands. There was no history of similar rash in any of his occupational colleagues or family members. He was also being stigmatized by his family and friends who were scared that they would be infected by making physical contact with him. He also could not continue with his regular farm work due to the debilitating nature of the disease and stigmatization. 
Physical examination done revealed some fresh but mostly healed papules with areas of lichenification and post-inflammatory hyperpigmentation on the extremities, buttocks and trunk. There were firm nodules on both sacral areas. The patient had bilateral inguinal and femoral lymphadenopathy with resultant hanging groin.

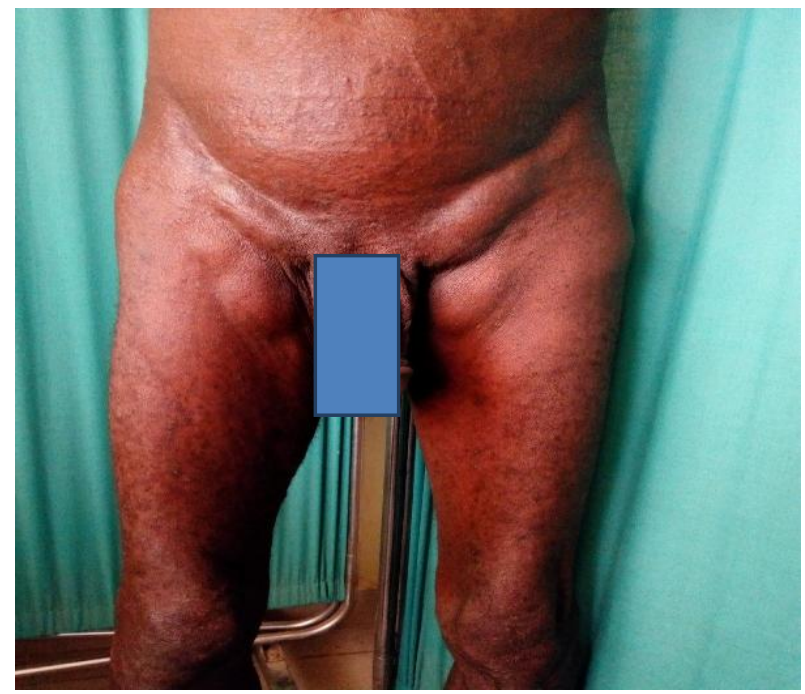

Figure 1: Bilateral inguinal and femoral lymphadenopathy (hanging groin)

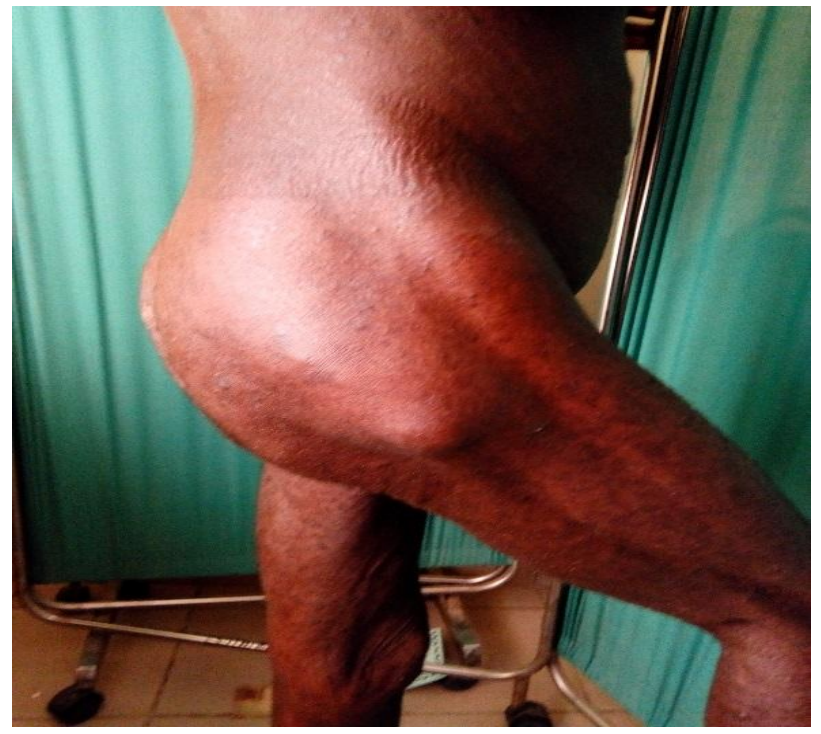

Figure 2: Onchocercal nodule in the sacral area

There were few areas on the skin of the trunk and extremities without any inflammation but had numerous scratch marks on account of the severe pruritus. He also had a reduction in his muscle bulk. He had no visual abnormalities and there were no abnormalities detected from the examination of other systems (Figs 1 and 2). He had sought care at various herbal homes and hospitals with no relief, but rather had worsening of symptoms which necessitated his presentation at the Missionary hospital from where he was referred. He did laboratory investigations which included a Full Blood Count that revealed eosinophilia and a skin snip taken from his iliac crest which revealed Microfilariae.

He was then placed on the following medications: Tabs ivermectin $12 \mathrm{mg}$ stat, then every 6 months for 10 years, Tabs albendazole 400mg stat, Caps doxycycline $100 \mathrm{mg}$ bd for 6 weeks, Tabs loratadine $10 \mathrm{mg}$ daily for 2 weeks and Emollients. He was referred also to the Ophthalmology clinic for further evaluation and also the Epidemiology Unit of the State to get the ivermectin tablets which was being given free to patients but at yearly intervals. He came back to the clinic after a 2 week appointment with marked resolution of the pruritus and some improvement in the skin lesions.

\section{Discussion}

Onchocerciasis is a very severe and debilitating parasitic infection of global concern. The prevalence and the magnitude of associated socio-economic effects vary in the different countries where the disease occurs. ${ }^{4}$ Worldwide, about 90 million people are at risk of which 17.6 million are infected, including 326,000 people who have gone blind, in 34 countries of the world. ${ }^{5}$ In the African continent, home to over $96 \%$ of all global cases, it has been reported in 26 countries including Nigeria. ${ }^{4}$

Onchocerciasis is one of the most studied filarial infections in Nigeria. It is estimated from previous studies that about 7-10 million Nigerians are infected with Onchocerca volvulus. There are approximately 40 million people who are at risk of the disease ${ }^{6}$ with many thousands suffering from disabling complications of the disease. ${ }^{7}$ Also, new foci of the infection are still being discovered and therefore its distribution could be far more extensive than has been earlier assumed. ${ }^{6}$ The classical skin lesions of onchocerciasias are a triad of pruritus, dermatitis and nodules (onchocercomas). ${ }^{8}$ The dermatitis is often variable in appearance depending on the chronicity of infection, age of the patient, geographic area where acquired and relative immune responsiveness. The index patient had all the three features. The eruptions (dermatitis) had features of chronicity with relative sparing of the face which is in keeping with onchocercal infections of forest Africa. 
He did not complain of any form of visual impairment but was still referred to see the ophthalmologist for complete evaluation prior to commencement of therapy. This was to rule out any of the ocular complications of onchocerciasis such as chorio-retinal changes or posterior segment disease. ${ }^{9}$ The treatment of onchocerciasis is aimed at the following: eliminating the worms, control of pruritus, reducing skin inflammation and surgical interventions such as nodulectomy. ${ }^{8}$ Ivermectin and diethylcarbamazine have been shown to have microfilaricidal properties and have been used extensively for therapy. However, the latter has a worse side effect profile and could lead to a paradoxical worsening of the symptoms referred to as the Mazotti reaction. It could also provoke visual loss in some patients. ${ }^{10}$ Doxycycline has activity against Wolbachia, an endosymbiont and also has some macrofilaricidal properties. ${ }^{11}$ Albendazole also has macrofilaricidal properties and can be used as an adjunct. $^{12}$ All the medications listed above except diethylcarbamazine were given to the patient as it has been shown from previous studies that these drugs have synergistic effects. ${ }^{11,13}$ The importance of making a correct and early diagnosis cannot be over emphasized as this patient's condition kept worsening due to ignorance and lack of medical expertise at his locale which further plunged him into more poverty and ostracism. The clinical response to treatment after 2 weeks is quite notable and he would have fared much better if had he presented earlier.

There are various ongoing efforts by the Federal Ministry of Health of Nigeria at Onchocerciasis elimination and recent data from the Nigeria Onchocerciasis Elimination: States classification 2016 showed that 8 states, including Benue state, has ongoing transmission of the infection. ${ }^{14}$ The above case report highlights the plight of one of the numerous people in the community who have chronic onchocerciasis and has been coping with the condition as well the numerous clinical and socio-economic complications.

There is therefore an urgent need to increase surveillance so that cases would be detected early especially in states where elimination has not been achieved. There is also need for community awareness about the disease especially among farmers and people who live near fast flowing rivers in rural communities. It is also pertinent that all efforts should be made by the stakeholders and agencies that fund the treatment of onchocerciasis with ivermectin that the medication should be given every 6 months rather than annually considering that there is possible ongoing transmission as studies have shown the benefits of the former over the latter. ${ }^{10}$

\section{Reference}

1. Centers for Disease Control and Prevention. Onchocerciasis General Information;2017.(site accessed 15/07/2018)

2. World Health Organization. WHO revised onchocerciasis guidelines as countries approach elimination targets;2017. (site accessed 15/07/2018)

3. Abiose A. Onchocercal eye disease and the impact of Mectizan treatment. Ann Trop Med Parasitol. 1998 ;92 Suppl 1:S11-22.

4. World Health Organization. WHO Expert Committee Onchocerciasis, Third report,Geneva.1987; Tech Rep Ser 752

5. World Health Organization. Geneva: WHO; 1990. World Report on Tropical Diseases.

6. World Health Organization. The Weekly Epidemiological Records, 71. Geneva: WHO; Onchocerciasis.

7. Anosike JC, Onwuliri COE. Studies on filariasis in Bauchi State, Nigeria 1. Endemicity of human onchocerciasis in Ningi Local Government Area. Ann Trop Med Parasitol. 1995;89(1):31-8.

8. James WD, Berger TG, Elston DM, Neuhaus IM.Andrew's Diseases of the Skin. Philadelphia, PA: Elsevier.2016

9. Newland HS, White AT, Greene BM, Murphy RP, Taylor HR. Ocular manifestations of onchocerciasis in a rain forest area of west africa. Br J Ophthalmol 1991;75:163-9.

10. Greene BM, Taylor HR, Cupp EW, Murphy RP et al. Comparison of Ivermectin and Diethylcarbamazine in the Treatment of Onchocerciasis. N Engl J Med 1985;313:133-8

11. Hoerauf A. Mand S, Adjei O, Fleischer B, Buttner DW. Depletion of Wolbachia endobacteria in Onchocerca volvulus by doxycycline and microfilaridermia after ivermectin treatment. The lancet 2001;357:1415-6

12. Cline BL, Hernandez JL, Mather FJ, Batholomew R, De Maza SN et al. Albendazole in the treatment of onchocerciasis: double-blind clinical trial in Venezuela. Am J Trop Med Hyg 1992;47:12-20.

13. Stingl P. Onchocerciasis: Developments in diagnosis, treatment and control. Int J Dermatol 2009;48:393-6.

14. Federal Ministry of Health of Nigeria. Nigeria Onchocer ciasis Elimination Plan 2017. 